\title{
Cash Management for Index Tracking
}

\author{
Gregory Connor and Hayne Leland
}

\begin{abstract}
Although all standard equity indexes have a zero weight in cash, managers running index-tracking portfolios often find that maintaining a positive cash holding is cost-efficient. This practice saves significantly on transaction costs because temporary cash inflows and outflows can be absorbed into the cash inventory. In this paper, a mean-variance framework is used to analyze the cash management problem for an index-tracking portfolio. The theoretical model is based on passive index tracking, but it has implications for any portfolio manager with an equity benchmark and random cash inflows and outflows.
\end{abstract}

A positive cash position has two disadvantages for an index-tracking portfolio (a portfolio designed to mimic the return on an equity index). First, equity indexes have a zero weight in cash, so a tracking portfolio with a positive weight will suffer from tracking error because of the mismatch in the cash weight. Second, the risk-free return is lower than the expected return on equities, so on average, a tracking portfolio with a positive cash holding will underperform the index portfolio.

The advantage of a positive cash position is that it lowers expected transaction costs. The per dollar transaction costs of equities are much higher than those of cash instruments. Time variation in cash inflows and outflows (e.g., from dividends, rights issues, new subscriptions, and fund redemptions) generates large equity transaction costs unless the manager holds a positive cash position on average.

The model presented in this article solves for the optimal cash-management policy of an index-tracking portfolio using a mean-variance framework. A portfolio manager's cost function at each date is assumed to be a linear combination of tracking error variance, current transaction costs, and the expected return on the portfolio. The tracking portfolio is subject to random cash inflows or outflows at each date. The manager chooses a cash inventory policy to minimize the discounted sum of expected cost over time.

The optimal cash-management policy has a simple form. The portfolio's cash weight as a proportion

Gregory Connor is director of research, Europe, for BARRA International and a visiting fellow at the London School of Economics. Hayne Leland is the Arno Rayner Professor of Finance in the Haas School of Business at the University of California, Berkeley. of portfolio value has an upper limit. If a cash inflow causes the cash weight to rise above this bound, the manager uses the extra cash to buy equities, forcing the cash weight back to the upper limit. Between the upper limit and zero, the cash weight is allowed to vary without any induced trading of equities. Because we assume that the manager cannot borrow, the manager's lower limit on the cash weight is zero. If a cash outflow causes the cash weight to fall temporarily below zero, the manager sells enough equities to push the weight back to zero.

This theoretical model is strictly applicable only to the case of an equity manager using a passive index-tracking strategy. We believe, however, that the model has relevance for any portfolio manager using an equity or fixed-income benchmark and having random cash inflows and outflows. As long as the manager's chosen strategy involves reasonably low turnover, and the manager does not hold cash for market-timing reasons, then our model should give a useful approximation to the optimal cash policy.

\section{THE CASH-MANAGEMENT PROBLEM}

Consider the problem of a manager attempting to track an equity index using a combination of cash and the equity index. The equity index has a zero position in cash, and the manager could guarantee exact tracking by setting the cash component of the portfolio to zero. The manager chooses to maintain a positive cash balance, however, to lower the transaction costs associated with frequently buying and selling the equity index in response to temporary cash inflows and outflows.

We assume that the cash inflows and outflows to the portfolio (dividends, rights offerings, fund redemptions, new fund subscriptions) are randomly 
distributed through time and proportional to the total value of the portfolio. Let $\omega_{t}$ denote the chosen cash weight at time $t$, and let $d_{t}$ denote the cash inflow (a negative value of $d_{t}$ denotes a cash outflow). Transactions in the cash account are costless, whereas transactions in the equity index are costly. The proportional transaction cost is the same for buying and selling of the index, and therefore, the time $t$ transaction cost is $c\left|d_{t}+\omega_{t-1}-\omega_{t}\right|$, where $|\bullet|$ denotes the absolute value and $c$ is the proportional transaction cost on trading in the equity index. ${ }^{1}$ We assume that the manager is restricted from borrowing, so the chosen cash weight, $\omega_{t}$, cannot be negative.

Let $\pi$ denote the expected return to the equity index in excess of the return to the risk-free asset. The decrease in expected return from holding a portfolio with cash weight $\omega_{t}$ (rather than holding the index portfolio) is $\omega t \pi$. Let $\sigma_{e}{ }^{2}$ denote the variance of the return of the equity index; the tracking-error variance of a portfolio with cash weight $\omega_{t}$ is $\omega_{t}{ }^{2} \sigma_{e}{ }^{2}$.

We assume that the manager's cost function at time $t, u_{t}$, is a linear combination of the portfolio's tracking-error variance, transaction costs, and expected excess return; that is,

$$
u\left(\omega_{t,} \omega_{t-1}\right)=\omega_{t} \pi+\lambda \omega_{t}^{2} \sigma_{e}^{2}+c\left|d_{t}+\omega_{t-1}-\omega_{t}\right| .
$$

The coefficient $\lambda$ captures the manager's risk aversion to tracking error.

This model is easy to apply in practice. Most of the required inputs are standard ones, familiar.to any investment manager running an index-tracking fund. The investment manager must decide on the parameter $\lambda$, based on the chosen trade-off between tracking-error variance and transaction cost. The expected excess return to the market index, $\pi$, determines the expected "cash drag" from underweighting equities in favor of cash. ${ }^{2}$ The volatility of the equity index, $\sigma_{e}{ }^{2}$, determines how much tracking-error variance is generated by a given amount of underweighting of equities. The only input that is not completely standard is the forecasting model for the random cash inflows and outflows to the portfolio. If we assume that these random shocks are normally distributed, then they are characterized by a mean $\mu_{d}$ and variance $\sigma_{d}^{2}$. These two parameters can be calibrated by estimating the per period average cash inflow into the account and the per period volatility of this cash inflow.

Let $r$ denote the manager's intertemporal discount rate. For simplicity, this rate can be set equal to the risk-free interest rate. The manager's objective is to choose a cash-management policy that minimizes the discounted sum of expected cost over an infinite planning horizon. Let $V\left(\omega_{t}\right)$ denote this discounted sum, given the current cash weight, $\omega_{t}$, and given that the manager uses the optimal policy in the future. The discounted sum of expected cost is

$$
V\left(\omega_{t}\right)=\sum_{s=1}^{\infty} \exp (-r s) E\left[u\left(\omega_{t+s}, \omega_{t+s-1}\right) \mid \omega_{t}\right]
$$

where $\exp (\bullet)$ denotes the exponential function. Determining the optimal cash policy is a stationary discounted dynamic-programming problem. The next section describes a solution to this problem for a continuous-time model with normally distributed cash inflows.

\section{A CONTINUOUS-TIME SOLUTION}

In a related paper, we derived a simple and easily implemented solution to the cash-management problem for the special case of a continuous-time model with constant parameters. ${ }^{3}$ Here, without going through the technical derivation, we briefly describe the key results from that paper and present the formula for the optimal cash policy.

Let a unit of time be fixed; we set one unit of time equal to one year. We let the length of the time period in the discrete-time model approach zero while holding the underlying parameters of the model constant. The discrete time period in the model is $\Delta t$ long, so that there are $1 / \Delta t$ time periods a year. The stock index has per period mean excess return of $\pi \Delta t$ and per period return variance of $\sigma_{e}^{2} \Delta t$. The manager's time discount rate is $r \Delta t$. The random cash inflow, $d_{t}$, is an independently and identically distributed normal random variable with mean $\mu_{\mathrm{d}} t \Delta$ and variance $\sigma d^{2} \Delta t$. If time is divided very finely (i.e., $\Delta t$ is very small), then we may approximate the value function, $V\left(\omega_{t+1}\right)$, using a Taylor series expansion around $\omega_{t}$, giving

$$
\begin{aligned}
V\left(\omega_{t+1}\right) & \approx V\left(\omega_{t}\right)+\left(\omega_{t+1}-\omega_{t}\right) V^{\prime}\left(\omega_{t}\right) \\
& +(1 / 2)\left(\omega_{t+1}-\omega_{t}\right)^{2} V^{\prime \prime}\left(\omega_{t}\right) .
\end{aligned}
$$

The optimal cash-management policy has a simple form. The cash position has an upper limit, denoted by $L$. If $\omega_{t-1}+d_{t}>L$, then the manager decreases the cash position to $L$. The manager increases the cash weight only if $\omega_{t}-1+d_{t}<0$, in which case the manager increases the cash weight to 0 . If $0 \leq \omega_{t-1}+$ $d_{t} \leq L$, then the manager does not rebalance. The optimization problem simplifies to finding the optimal value for $L$. The second-order approximation (1) generates a second-order differential equation defining $V$. Solving this differential equation subject to the appropriate boundary conditions and then optimizing gives the following implicit equation for $L$ :

$A \bullet \exp (B \bullet L)+(C+D \bullet L) \bullet \exp (E \bullet L)+F \bullet L \bullet \exp (G \bullet L)=0 .(2)$

The coefficients $A, B, C, D, E, F$, and $G$ are functions of the model parameters shown below. To simplify 
notation, define $z=\left(\mu_{d}^{2}+2 r \sigma_{d}^{2}\right)^{1 / 2} / \sigma_{d}^{2}$ and $x=-\mu_{d} / \sigma_{d}^{2}$. The values of $A$ through $G$ are given by

$$
\begin{aligned}
& \mathrm{A}=(x-z)\left[\left(\lambda \sigma_{\mathrm{e}}{ }^{2} / r\right)\left(2 \mu_{d} / r\right)+\pi / r-c\right]-2 \lambda \sigma_{e}{ }^{2} / r \\
& B=x-z \\
& C=(x+z)\left[c-\left(\lambda \sigma_{e}{ }^{2} / r\right)\left(2 \mu_{d} / r\right)+\pi / r\right]+2 \lambda \sigma_{e}{ }^{2} / r \\
& D=x+z \\
& E=2 z\left[c+\left(\lambda \sigma_{e}{ }^{2} / r\right)\left(2 \mu_{d} / r\right)+\pi / r\right] \\
& F=2 x \\
& G=\left(2 \lambda \sigma_{e}{ }^{2} / r\right)(x-z)
\end{aligned}
$$

For any particular values of the model parameters, $\lambda, \mu_{\mathrm{d}}, \sigma_{d}{ }^{2}, \sigma_{\mathrm{e}}{ }^{2}, c, r$, and $\pi$, Equation (2) can be solved for $L$. Once the optimal $L$ is found, it is also possible to solve for the expected portfolio turnover associated with $L$.

Table 1 shows optimal cash inventory policies and associated expected annual turnover under a variety of assumptions about the parameters. We chose $\lambda=10, r=4$ percent, $\pi=6$ percent, $\sigma_{e}=20$ percent, $\mu_{d}=0$, and $\sigma_{d}=10$ percent as the base case values of the parameters. Each panel of the table shows the solution as we perturbed one of the parameters above and below its base case value. We tried to cover what we believed to be a "reasonable range" of values; the perturbation values are not always symmetric around the base case value.

For each of the parameters, the optimal policy varies in the intuitively reasonable direction as the parameter value varies. In particular, the upper limit on cash increases as the proportional transaction cost increases, cash inflow volatility increases, expected cash inflow decreases, equity risk premium decreases, market index volatility decreases, and the risk-aversion parameter decreases. Figure 1 graphs the upper limit on cash and expected turnover over the same range of parameter values as in Table 1, but with a finer grid between values (the range between the high and the low in Table 1 is divided into 100 equally spaced points).

The optimal policy is particularly sensitive to the proportional transaction cost, $c$, and the cash inflow volatility, $\sigma_{d}$. It is moderately sensitive to the ex-

\begin{tabular}{|c|c|c|c|c|c|c|c|}
\hline \multicolumn{8}{|c|}{ Base case: $c=1 \%, \mu_{d}=0, \sigma_{d}=10 \%, \pi=6 \%, \sigma_{e}=20 \%, \lambda=10, r=4 \%$} \\
\hline $\begin{array}{c}\text { Cash } \\
\text { Limit (\%) }\end{array}$ & $\begin{array}{c}\text { Expected } \\
\text { Annual } \\
\text { Turnover (\%) }\end{array}$ & & & & & & \\
\hline 4.84 & 20.71 & & & & & & \\
\hline \multicolumn{8}{|c|}{ Perturbed cases: Same values as in the base case, except as shown } \\
\hline $\begin{array}{c}\text { Cash } \\
\text { Limit(\%) }\end{array}$ & $\begin{array}{c}\text { Expected } \\
\text { Annual } \\
\text { Turnover (\%) }\end{array}$ & $\begin{array}{c}\text { Cash } \\
\text { Limit (\%) }\end{array}$ & $\begin{array}{c}\text { Expected } \\
\text { Annual } \\
\text { Turnover }(\%)\end{array}$ & $\begin{array}{c}\text { Cash } \\
\text { Limit (\%) }\end{array}$ & $\begin{array}{c}\text { Expected } \\
\text { Annual } \\
\text { Turnover (\%) }\end{array}$ & $\begin{array}{c}\text { Cash } \\
\text { Limit (\%) }\end{array}$ & $\begin{array}{c}\text { Expected } \\
\text { Annual } \\
\text { Turnover (\%) }\end{array}$ \\
\hline \multicolumn{2}{|c|}{$c=0.1 \%$} & \multicolumn{2}{|c|}{$c=0.5 \%$} & \multicolumn{2}{|c|}{$c=5 \%$} & \multicolumn{2}{|c|}{$c=10 \%$} \\
\hline 1.70 & 58.77 & 3.56 & 28.11 & 9.55 & 10.54 & 12.65 & 7.99 \\
\hline \multicolumn{2}{|c|}{$\mu_{d}=-10 \%$} & \multicolumn{2}{|c|}{$\mu_{d}=-5 \%$} & \multicolumn{2}{|c|}{$\mu_{d}=5 \%$} & \multicolumn{2}{|c|}{$\mu_{d}=10 \%$} \\
\hline 5.64 & 19.63 & 5.21 & 19.66 & 4.51 & 22.58 & 4.22 & 25.10 \\
\hline \multicolumn{2}{|c|}{$\sigma_{d}=1 \%$} & \multicolumn{2}{|c|}{$\sigma_{d}=5 \%$} & \multicolumn{2}{|c|}{$\sigma_{d}=15 \%$} & \multicolumn{2}{|c|}{$\sigma_{d}=20 \%$} \\
\hline 0.56 & 1.77 & 2.61 & 9.61 & 6.84 & 32.95 & 8.68 & 46.12 \\
\hline \multicolumn{2}{|c|}{$\pi=0$} & \multicolumn{2}{|c|}{$\pi=5 \%$} & \multicolumn{2}{|c|}{$\pi=10 \%$} & \multicolumn{2}{|c|}{$\pi=20 \%$} \\
\hline 7.23 & 13.89 & 5.10 & 19.65 & 4.06 & 24.66 & 3.04 & 32.88 \\
\hline \multicolumn{2}{|c|}{$\sigma_{e}=10 \%$} & \multicolumn{2}{|c|}{$\sigma_{e}=15 \%$} & \multicolumn{2}{|c|}{$\sigma_{e}=40 \%$} & \multicolumn{2}{|c|}{$\sigma_{e}=50 \%$} \\
\hline 5.46 & 18.34 & 5.16 & 19.43 & 3.78 & 26.51 & 3.40 & 29.44 \\
\hline \multicolumn{2}{|c|}{$\lambda=0$} & \multicolumn{2}{|c|}{$\lambda=5$} & \multicolumn{2}{|c|}{$\lambda=15$} & \multicolumn{2}{|c|}{$\lambda=40$} \\
\hline 5.79 & 17.32 & 5.21 & 19.22 & 4.56 & 21.96 & 3.78 & 26.51 \\
\hline
\end{tabular}
pected average cash inflow, $\mu_{d}$, and the equity risk premium, $\pi$. It is less sensitive to the risk-aversion parameter, $\lambda$, and the volatility of the equity index, $\sigma_{e}$ at least over a range of parameter values that we consider "reasonable."

Table 1. Optimal Cash Policies and the Associated Expected Turnover 
Figure 1. Optimal Cash Limit and Expected Turnover for Varying Parameter Values
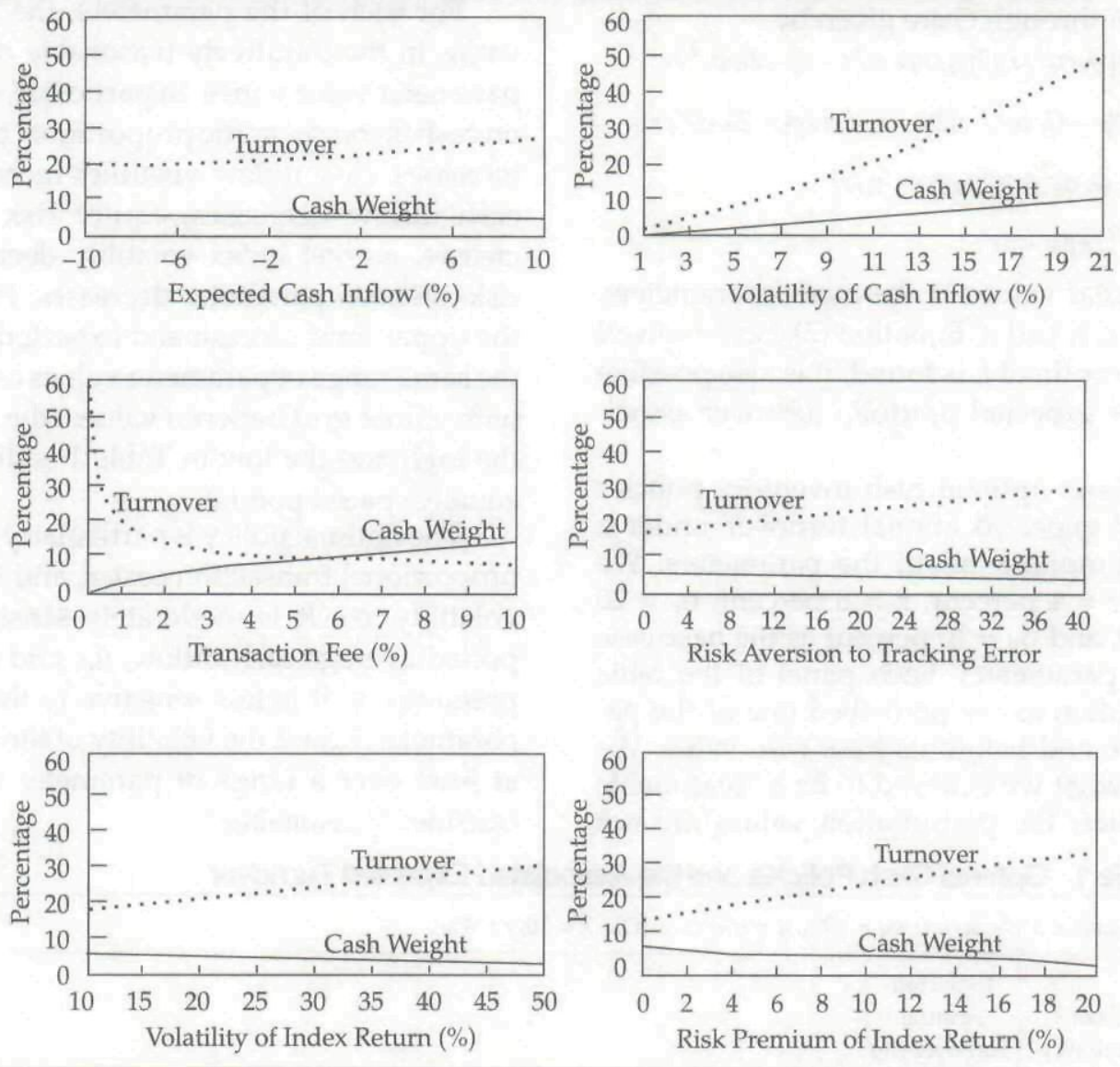

\section{APPLYING THE CASH-MANAGEMENT MODEL WTHOUT FULL REPLICATION}

The cash-management model assumes that within the equity component of the tracking portfolio, the manager exactly mimics the index weights, a strategy known as full replication. In some situations, such as tracking standard equity indexes in developed capital markets, full replication is the most common procedure. In other situations, for example, index tracking in emerging markets, many managers choose to weight individual equities differently from the index weights. For instance, many emerging market managers limit the number of assets in the tracking portfolio to 15 to 30 securities, although the actual index consists of 300 or more securities.

We are not able to solve the cash-management problem with complete generality in the absence of full replication. The dynamic optimization problem becomes very complex. Our dynamic model has one choice variable, which is the proportion of the portfolio in cash. Suppose that the equity index portfolio has $n$ individual securities. Without full replication, the number of choice variables increases to $n$ : the proportions of the portfolio in cash and in each of the equity securities, minus 1 . The structure of the opti- mization problem becomes very complicated; the behavior of each individual security through time affects the optimal dynamic portfolio weight for every other security, including but not limited to the cash weight.

Take as given the problem of choosing a basket of equities to optimally track the index. ${ }^{4}$ Our model, with slight modifications, can then be used to solve the problem of mixing this preselected equity portfolio with an optimal proportion of cash. The definitions of the inputs to the model change, but the basic form of the optimization problem is the same.

As before, let $\omega_{t}$ denote the proportion of the portfolio in cash and $\left(1-\omega_{t}\right)$ denote the proportion in the chosen equity portfolio (not necessarily the index portfolio). Let $\rho$ denote the correlation between the chosen equity portfolio and the index portfolio. The chosen equity portfolio and the index portfolio are assumed to have equal return variance, $\sigma_{e}{ }^{2}$, and equal expected excess return, $\pi$. Note that the expected excess return to the tracking portfolio with cash weight $\omega_{t}$ is (as before) $\left(1-\omega_{t}\right) \pi$. It is easy to show that the tracking-error variance is $2 \sigma_{e}^{2}\left(1-\omega_{t}+\right.$ $\left.\rho \omega_{t}-\rho+\omega_{t}^{2}\right)$. The term $2 \sigma_{e}^{2}(1-\rho)$ in the trackingerror variance can be omitted from the minimization problem because it does not depend upon $\omega_{t}$ and 
therefore does not affect the optimization over $\omega_{t}$. We assume, as before, that there is a proportional transaction cost of $c$ for buying or selling the equity portfolio. The cost function is the same as in the case of full replication. Collecting the expressions for each of the terms and inserting them into the cost function gives

$u_{t}=\omega_{t} \pi+\lambda \omega_{t}^{2} \sigma_{e}^{2}+2 \lambda(1-\rho) \omega_{t} \sigma_{e}^{2}+c\left|\omega_{t}-\omega_{t, t-1}\right|$.

With one change to the definitions of the inputs, the optimization problem has the same structure as in the case of full replication. Simply replacing $\pi$ with $\pi^{*}=\pi+2 \lambda(1-\rho) \sigma_{e}{ }^{2}$, the same model as before can be used to solve for the optimal cash-management policy. Table 2 shows the results with the correlation coefficient, $\rho$, set equal to 1.0 (the base case), 0.98 , $0.90,0.80$, and 0.70 . The imperfect correlation between the equity-only part of the portfolio and the index portfolio lowers the optimal cash limit. The expected turnover is also shown, but it does not include the turnover that is, in practice, necessary to maintain the tracking performance of the equity-only part of the portfolio. The model assumes that the manager can maintain a fixed correlation of the equity portfolio and the index portfolio without any turnover.

In the case of active strategies, in which the manager dynamically varies the components of the equity portfolio for stock selection purposes or the equity/cash mix for market-timing purposes, the cash-management model does not strictly apply. Nevertheless, an active manager can view the model as a useful first approximation to determining the correct cash holding. Solving for the truly optimal cash-management policy associated with an active equity portfolio strategy goes beyond the scope of this article.

\section{SUMMARY}

The two disadvantages of a positive cash holding for an index-tracking manager are that it increases tracking error, and because cash has a lower expected return than equities, it reduces the expected return of the portfolio. The one advantage of a positive cash holding is that it reduces the transaction costs generated by transitory cash inflows and outflows to the portfolio. We used a mean-variance objective function to balance these three influences (tracking error, expected return, and transaction cost) in order to analyze the optimal cash policy.

The optimal cash policy has a simple form. The cash weight as a proportion of portfolio value has an upper limit. If the portfolio's cash weight drifts above this upper limit, the manager buys more of the equity index and so forces the cash weight back down to the upper limit. As long as the cash weight stays between this maximum and zero, the manager does not rebalance the position. If the cash weight falls below zero, the manager is forced to sell some of the holding in the equity index to avoid violating a no-borrowing constraint.

Our cash-management model is strictly applicable only to a manager running a passive index-tracking strategy, but we believe that our findings are of some relevance for any portfolio manager measured against an equity benchmark, using a reasonably low turnover strategy and not holding cash for markettiming purposes.

Table 2. Optimal Cash Policies and Expected Turnover with Imperfect Tracking

\begin{tabular}{|c|c|c|c|c|c|c|c|}
\hline \multicolumn{8}{|c|}{ Base case: $\rho=1, c=1 \%, \mu_{d}=0, \sigma_{d}=10 \%, \pi=6 \%, \sigma_{e}=20 \%, \lambda=10, r=4 \%$} \\
\hline $\begin{array}{c}\text { Cash } \\
\text { Limit (\%) }\end{array}$ & $\begin{array}{c}\text { Expected } \\
\text { Annual } \\
\text { Turnover }(\%)\end{array}$ & & & & & & \\
\hline 4.84 & 20.71 & & & & & & \\
\hline \multicolumn{8}{|c|}{ Perturbed cases: Same values as in the base case, except as shown } \\
\hline $\begin{array}{c}\text { Cash } \\
\text { Limit (\%) }\end{array}$ & $\begin{array}{c}\text { Expected } \\
\text { Annual } \\
\text { Turnover }(\%)\end{array}$ & $\begin{array}{c}\text { Cash } \\
\text { Limit (\%) }\end{array}$ & $\begin{array}{c}\text { Expected } \\
\text { Annual } \\
\text { Turnover (\%) }\end{array}$ & $\begin{array}{c}\text { Cash } \\
\text { Limit (\%) }\end{array}$ & $\begin{array}{c}\text { Expected } \\
\text { Annual } \\
\text { Turnover }(\%)\end{array}$ & $\begin{array}{c}\text { Cash } \\
\text { Limit (\%) }\end{array}$ & $\begin{array}{c}\text { Expected } \\
\text { Annual } \\
\text { Turnover }(\%)\end{array}$ \\
\hline \multicolumn{2}{|c|}{$\rho=0.98$} & \multicolumn{2}{|c|}{$\rho=0.90$} & \multicolumn{2}{|c|}{$\rho=0.80$} & \multicolumn{2}{|c|}{$\rho=0.70$} \\
\hline 4.48 & 22.35 & 3.55 & 28.19 & 2.92 & 34.32 & 2.53 & 39.60 \\
\hline
\end{tabular}




\section{NOTES}

1. To simplify the model, we assume that the transaction costs are paid separately by the manager as a "fee" from external income. They appear in the manager's objective function but do not directly decrease the value of the assets under management.

2. We are assuming that the index fund manager suffers from the lower return associated with cash but does not benefit from the lower risk associated with holding cash rather than holding the index portfolio. Our model assumes that the manager is judged on his return performance relative to the equity index and that the performance benchmark is not adjusted downward if the manager chooses to hold more cash.

3. See Hayne Leland and Gregory Connor, "Optimal Cash Management for Investment Funds," Working Paper no. 244, Institute of Business and Economic Research, University of Califor- nia, Berkeley (1995)

4. See Stanley R. Pliska, "Index Arbitrage: Choosing Minimum Variance Basket Trading Opportunities," Options: Recent Advances in Theory and Practice, Stewart Hodges, ed., Manchester: Manchester University Press (1990), for one model of how to construct an index-tracking portfolio using only a subset of the equities in the index. Pliska assumes that transaction costs are zero and that the chosen subset of equities is given exogenously.

5. We would like to thank Ian Buckley, Lucie Chaumeton, and Ross Curds for excellent research assistance, and Fischer Black, Ron Kahn, and John Remmert for helpful discussions. Any remaining errors or omissions are our responsibility. 
Copyright of Financial Analysts Journal is the property of CFA Institute. The copyright in an individual article may be maintained by the author in certain cases. Content may not be copied or emailed to multiple sites or posted to a listserv without the copyright holder's express written permission. However, users may print, download, or email articles for individual use. 